\title{
Non Bianisotropic Complementary Split Ring Resonators as Angular Selective Metasurfaces
}

\author{
Pablo Rodríguez-Ulibarri ${ }^{1}$ and Miguel Beruete $1,2, *$ \\ ${ }^{1}$ Antennas Group - TERALAB, Public University of Navarra, Campus Arrosadía s/n 31006, Pamplona, Spain \\ ${ }^{2}$ Institute of Smart Cities, Public University of Navarra, Campus Arrosadía s/n 31006, Pamplona, Spain \\ ${ }^{*}$ Corresponding author: miguel.beruete@unavarra.es
}

Compiled June 8, 2017

\begin{abstract}
In this work, a metasurface with unconventional angular performance and composed by nonbianisotropic complementary split ring resonator (NB-CSRR) is analyzed numerically and experimentally. A numerical study comparing the performance with the original complementary split ring resonator is conducted showing very interesting transmission properties such as complete filtering under normal incidence and high transmission peaks at oblique incidence for the NB-CSRR metasurface. A bi-layered NB-CSRR prototype working at millimeter waves with a total thickness of 100 microns $(0.1$ times the operation wavelength) is fabricated and tested with a good agreement between simulation results and experiments. Very exciting applications based on the presented structure such as advanced angular selectivity devices with great rejection level at normal incidence and angular sensing devices can be envisaged. (c) 2017 Optical Society of America
\end{abstract}

OCIS codes: (160.3918) Metamaterials; (050.6624) Subwavelength structures.

http://dx.doi.org/10.1364/ao.XX.XXXXXX

\section{INTRODUCTION}

Since the advent of metamaterials at the beginning of this century, the split ring resonator (SRR) proposed by Pendry has been one of the major exponents for the realization of these artificial structures. The SRR is a subwavelength metallic ring element that exhibits a strong magnetic response, even though their constituent elements are non-magnetic. When multiple SRR elements are properly arranged into a periodic array, the effective permeability of the full structure can be negative in a certain frequency range [1]. This property led to the first experimental demonstration of negative refractive index media by combining a matrix of SRRs interspersed with metallic wires able to emulate an effective medium with negative permittivity [2]. This experiment corroborated the theoretical proposal of such kind of media developed by Veselago back in 1968 [3].

Soon after the seminal work of Pendry, SRRs received a great deal of attention from the scientific community giving rise to thorough investigations about their frequency response, equivalent circuit models, cross polarization effects and potential applications mainly as filters solution in planar technology [4-7]. A well-founded theoretical study of the physical grounds of these particles can be found in [4]. There, the bianisotropic character of the SRR was reported and the appearance of an electric response with a magnetic excitation (and vice versa) was corroborated. Several alternatives were proposed for avoiding bianisotropy such as etching each of the rings at opposite sides of the substrate and by connecting the internal and external rings with a metallic strip [6].

The complementary version of the SRR (called CSRR) was first reported by Falcone et al. in [8] by exploiting the Babinet's principle, strictly valid for infinitely thin and lossless metallic screens. In that work, the experimental demonstration of the bandstop/bandpass character of SRR/CSRR metasurfaces was also performed. Since then, SRRs and CSRRs have been widely used for filter applications and mostly analyzed in waveguides and planar circuit devices [6] as well as spatial filters in the metasurface versions $[7,9]$ and even as sensing platforms within the terahertz $(\mathrm{THz})$ regime [10].

Metasurfaces are the 2D evolution of metamaterials and lately an extensive research effort has been put on developing more compact structures to provide new routes for the control of electromagnetic waves [11, 12]. To name a few, beam steering by means of phase variation phenomena between adjacent elements [13], performing mathematical operations of the impinging wave [14], asymmetric transmission devices [15] and cloaking carpets [16] are some of the applications recently proposed that take advantage of the new possibilities offered by metasurfaces. In addition a recent interest has arisen for hyperbolic metamaterials which present unconventional equi-frequency diagrams that result into very sophisticated oblique incidence responses [17]. Metasurfaces based on asymmetric split resonators (ASR) have 
been used for wavefront manipulation and as ultrahigh angular selective structures working at $\mathrm{THz}[18,19]$.

In this work we follow this path and extend the reach of CSRR metasurfaces by using non-bianisotropic CSRR (NB-CSRR) elements $[6,20,21]$. This way, we obtain a non-conventional angular response, with normally incident waves experiencing high reflection and waves at oblique incidence being partially or even totally transmitted. This response is obtained for the two principal incidence planes endowing the structure with a polarization independent character. We perform a simulation study followed by an experimental demonstration at millimeterwaves. Mono and bi-layered configurations are numerically studied. Being the latter structure more interesting in terms of rejection of normal incidence waves, a prototype has been fabricated and tested. The results confirmed low transmission levels for normal incidence and high transmission at oblique elevation angles from $50^{\circ}$ to $70^{\circ}$.

The angular selectivity properties of the presented structure enables angular filtering applications such as isolating transmitting from receiving antennas in dual band frequency and spatial antennas systems. Nowadays multiple technologies are implemented in the same device so in occasions angular and frequency filtering properties need to be combined. In the presented structure, a strong contrast between normally and oblique incidence waves is obtained. In addition, the frequency response becomes broader as the angle of incidence increases. Therefore, structures with broadband transmission for large incidence angles and near total reflection for normal incidence can be envisaged. Another field where this structure may find application is sensing. In some situations oblique incidence may be required to avoid obstacles between the transmitter and the sample. Then a structure with high transmission under oblique incidence would be very useful. Besides, at large oblique angles an incident wave would travel larger distances inside the analyte enhancing the performance.

\section{NB-CSRR METASURFACES}

As explained in [8] CSRR elements (shown schematically in Fig. 1(b)) can be excited by an axial electric field or by a magnetic field parallel to the metallic strips between ring apertures, due to their bianisotropy property. This bianisotropy can be cancelled following several strategies, as discussed in [4]. A simple way is to connect the inner and outer ring apertures with two air bridges, leading to the so-called NB-CSRR shown schematically in Fig. 1(a), whose fundamental resonance can only be excited by an axial electric field. This implies that the new particle can only be excited by a transverse magnetic (TM) plane wave at oblique incidence, since it has the required longitudinal electric component $\left(E_{z}\right)$. In contrast, a CSRR can be excited at normal incidence, provided the magnetic field is parallel to the strips between the ring apertures and, under oblique incidence, it presents interesting cross-polarization effects [7]. In the following, we perform an exhaustive comparison between CSRR and NB-CSRR transmission results under normal and oblique incidence for transverse magnetic (TM) and transverse electric (TE) incidence considering both mono-layer and bi-layer structures.

\section{A. Mono-layer Structure}

Figure 1 shows the simulated transmission results for both CSRR and NB-CSRR structures at different angles of incidence $(\theta=$ $0^{\circ}, 30^{\circ}$ and $\left.60^{\circ}\right)$ under TE and TM polarization. Both principal incidence planes are considered, $x z\left(\phi=0^{\circ}\right)$ and $y z\left(\phi=90^{\circ}\right)$ [see

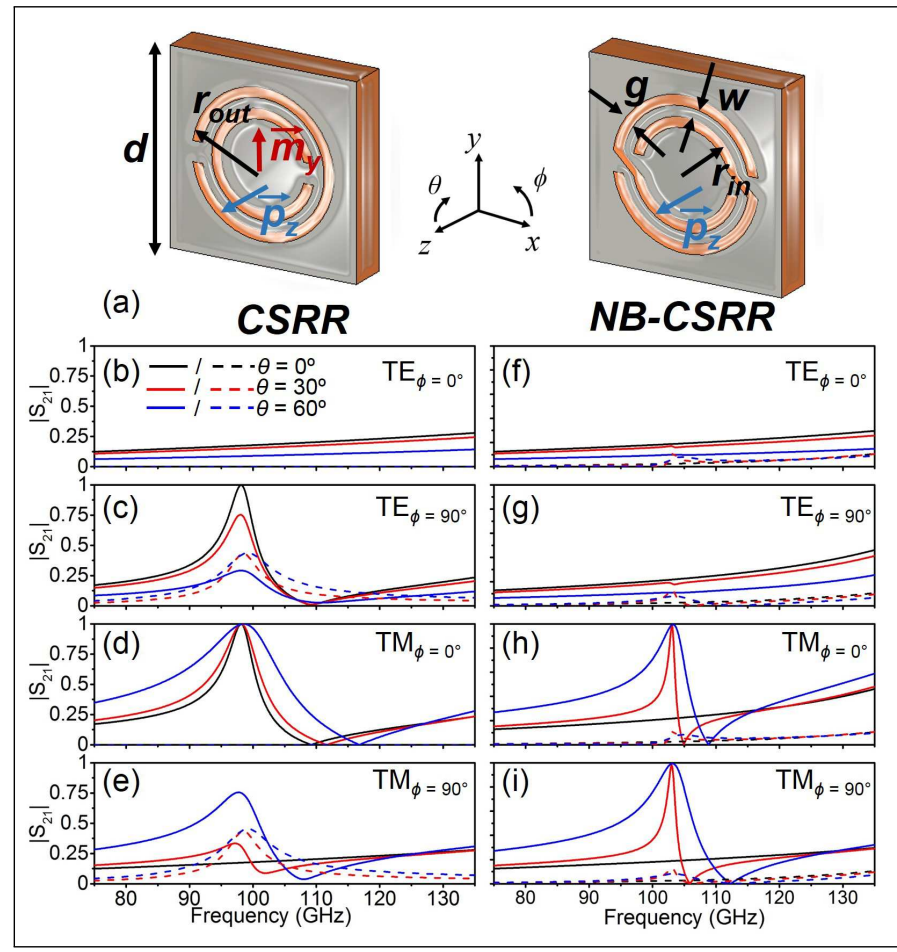

Fig. 1. (a) CSRR and NB-CSRR unit cell elements along with the electric and magnetic dipoles and the reference coordinate system. Geometrical parameters: lattice period $d=0.48 \mathrm{~mm}$; inner radius, $r_{i n}=0.12 \mathrm{~mm}$; outer radius, $r_{\text {out }}=0.17 \mathrm{~mm}$; air gap, $g=0.025 \mathrm{~mm}$ and metal gap, $w=0.025 \mathrm{~mm}$. (b)-(i) Transmission results of co-polar (solid line) and cross-polar (dashed line) components obtained at $\theta=0^{\circ}$ (black line), $\theta=30^{\circ}$ (red line) and $\theta=60^{\circ}$ (blue line). (b) CSRR - $\mathrm{TE}_{\phi=0^{\circ}}$. (c) CSRR - $\mathrm{TE}_{\phi=90^{\circ}}$. (d) CSRR - $\mathrm{TM}_{\phi=0^{\circ}}$. (e) CSRR - $\mathrm{TM}_{\phi=90^{\circ}}$. (f) NB-CSRR - $\mathrm{TE}_{\phi=0^{\circ}}$. (g) NB-CSRR - TE $E_{\phi=90^{\circ}}$. (h) NB-CSRR - TM $\mathrm{T}_{\phi=0^{\circ}}$. (i) NB-CSRR $\mathrm{TM}_{\phi=90^{\circ}}$.

Fig.1 (a)]. Simulations were carried out with the commercial fullwave numerical solver CST Microwave Studio ${ }^{\mathrm{TM}}$ with unit cell boundary conditions [22]. For this initial study, perfect electric conductor (PEC) material and a lossless dielectric substrate with relative permittivity $\varepsilon_{S}=2.35$ and height $h=0.1 \mathrm{~mm}$ were used for the metal and dielectric parts respectively. The geometrical parameters of the CSRR/NB-CSRR elements are depicted in Fig. 1(a) with their values given in the caption. Fig. 1(b)-(i) shows the transmission coefficient for the copolar (solid line) and crosspolar (dashed line) components of CSRR [Fig.1 (b)-(e)] and NB-CSRR [Fig.1 (f)-(i)] structures.

Let us first focus on the CSRR case. Under TE wave incidence in the $x z$-plane (labelled as $\mathrm{TE}_{\phi=0^{\circ}}$ ) the field components of the incident wave are $E_{y}, H_{x}$ and $H_{z}$. Therefore, the CSRR cannot be excited, given that none of these components is parallel to the resonant dipoles of the fundamental resonance $\left(p_{z}\right.$ or $\left.m_{y}\right)$. However, by switching to the $y z$-plane, i.e. $\mathrm{TE}_{\phi=90^{\circ}}$, the incident wave has an $H_{y}$ field component able to excite the tangent magnetic dipole, $m_{y}$, giving rise to a resonance peak at roughly 98 $\mathrm{GHz}$, even at normal incidence [see Fig. 1(c)]. When the angle of incidence, $\theta$, is changed to $30^{\circ}$ and $60^{\circ}$ the resonance peak still appears in the spectrum but with a reduced transmission level. This behavior is a direct consequence of the bianisotropy of the CSRR element as explained in $[5,7]$, that produces a cross 


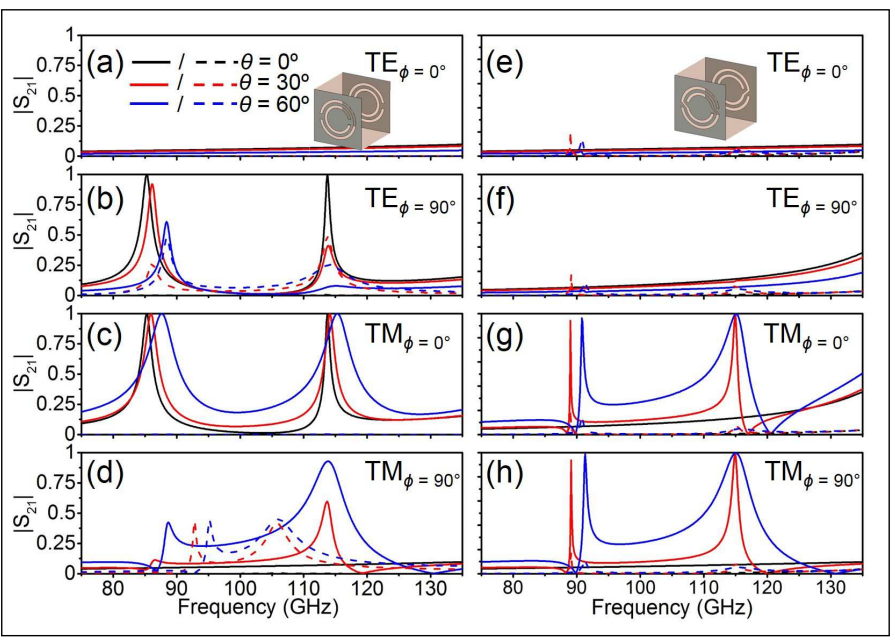

Fig. 2. Transmission results of co-polar (solid line) and crosspolar (dashed line) components obtained at $\theta=0^{\circ}$ (black line), $\theta=30^{\circ}$ (red line) and $\theta=60^{\circ}$ (blue line) for bi-layer configurations. (a) CSRR $-\mathrm{TE}_{\phi=0^{\circ}}$. (b) $\mathrm{CSRR}-\mathrm{TE}_{\phi=90^{\circ}}$. (c) CSRR $\mathrm{TM}_{\phi=0^{\circ}}$. (d) CSRR - $\mathrm{TM}_{\phi=90^{\circ}}$. (e) NB-CSRR - TE $\mathrm{T}_{\phi=0^{\circ}}$. (f) NBCSRR - TE T=90 $^{\circ}$. (h) NB-CSRR - TM $\mathrm{TM}_{\phi=0^{\circ}}$. (g) NB-CSRR - $\mathrm{TM}_{\phi=90^{\circ}}$. Insets: Bi-layered CSRR and NB-CSRR unit cells.

polarization peak even higher than the copolar component for $\theta=60^{\circ}$. For the $\mathrm{TM}_{\phi=0^{\circ}}$ case, the incident wave has $H_{y}, E_{x}$ and $E_{z}$. Therefore, the CSRR can be excited magnetically under normal incidence and both magnetically and electrically under oblique incidence. The joint excitation of both resonance dipoles leads to a larger bandwidth when the incidence angle is increased compared to the normal incidence case. For the $\mathrm{TM}_{\phi=90^{\circ}}$ case the only field component able to excite the CSRR is the axial electric field, $E_{z}$ and hence, a transmission peak appears only under oblique incidence. Cross polarization effects arise as well, due to the magnetic dipole excited through the electric dipole resonance. Looking at these results, it is noticed that cross-polarization is present only when the incidence is in the $y z$-plane. All these results are in good agreement with those reported in $[5,7]$.

The NB-CSRR element behaves quite differently from the CSRR. First, it is obvious that the cross polarization effects should be lower since bianisotropy is largely mitigated (practically eliminated) by the air connections between the inner and outer rings. Fig. 1 (f)-(i) shows that the cross-polarization level is quite low in all cases. This modification of the CSRR geometry cancels the tangent magnetic dipole. Therefore, the only way to excite the quasistatic resonance of this structure is under TM oblique incidence that has the required $E_{z}$ component parallel to the $p_{z}$ dipole. Fig. 1 (h) and (i) show that at normal incidence there is low transmission and there is not any signature of resonance effects. In contrast, when $\theta=30^{\circ}$ and $\theta=60^{\circ}$ a resonant transmission peak with almost unity transmission arises near $103 \mathrm{GHz}$, i.e. is slightly upshifted with respect to the CSRR case. From these results, the angular selectivity feature of the NB-CSRR metasurface is clearly demonstrated.

\section{B. Bi-layer Structure}

Next, we analyze the bi-layer structure shown in Fig. 2 insets, which is more interesting from a practical point of view. This structure shows a higher rejection at normal incidence which could be very attractive for angular selective devices. In addition,
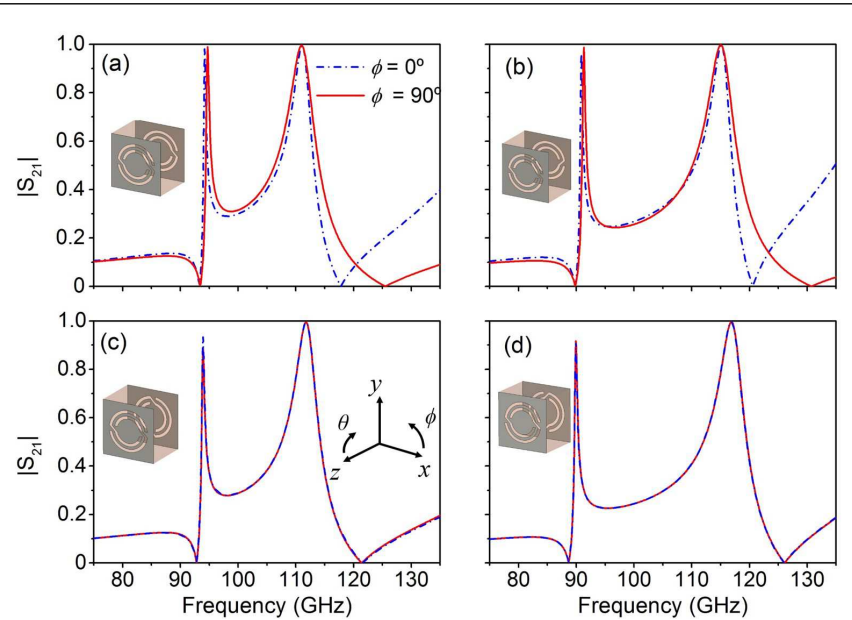

Fig. 3. Transmission results under different rotation scenarios. (a) $y$-Rotation $=0^{\circ}$; (b) $y$-Rotation $=180^{\circ}$; (c) $y$-Rotation $=180^{\circ}$ and $z$-rotation $=90^{\circ} ;(\mathrm{d}) y$-Rotation $=0^{\circ}$ and $z$-rotation $=90^{\circ}$. Curves in panels (c) and (d) are overlapped.

due to the coupling between layers, the resonance peak is split in two for both configurations [23, 24] adding another interesting functionality as double band device. The simulation results are shown in Fig. 2. As before, we compare bi-layer structures composed of CSRRs [Fig. 2(a)-(d)] or NB-CSRRs [Fig. 2(e)-(h)]. In general, the results follow the tendency shown in Fig. 1. The main difference is the splitting of the resonance peak in two due to the coupling of resonators between both layers (this will be studied in more detail later). Also, in contrast with the monolayer case, the cross-polarization curves for the $\mathrm{TE}_{\phi=90^{\circ}}$ and the $\mathrm{TM}_{\phi=90^{\circ}}$ are different due to the coupling between layers.

As observed in Fig. 2, the NB-CSRR metasurface has transmission bands only under oblique TM incidence, in good agreement with the results of Fig. 1. Again, the peak is split in two, and, as expected, the rejection level for normal incidence is enhanced due to the presence of the second metasurface. In addition the cross polarization levels are negligible in comparison with the copolar component. Due to practical reasons that will be addressed in the experimental section, there is a $180^{\circ}$ rotation (on $y$ axis) between patterned metasurface layers. This rotation between layers does not affect the overall performance of the structure. In fact, the NB-CSRR performance is quite robust to rotation of the second layer in both $y$ and $z$ axes. Figure 3 shows transmission results for bi-layer NB-CSRR structures under oblique incidence $\left(\theta=60^{\circ}\right)$ and different rotations of the outer particle in both principal polarization planes $\left(\phi=0^{\circ}\right.$ and $\left.\phi=90^{\circ}\right)$. As it can be seen, aside from a small frequency shift the transmission spectra are very similar for all cases. This is not the case for the classical CSRR where the results differ considerably if the second layer is rotated or not (not shown here). The reason is that the NB-CSRR has a single axial resonant electric dipole and therefore is less sensitive to a rotation of the elements and/or incidence plane.

The inclusion of an additional metasurface layer deserves some more insightful discussion. Figure 4 shows a parametric study varying the substrate height $(h)$, i.e. distance between both metallic layers. $\mathrm{TM}_{\phi=0^{\circ}}$ oblique incidence $\left(\theta=60^{\circ}\right)$ is assumed for the NB-CSRR structure. As mentioned above, due to the coupling between layers, the original transmission peak is split in two due to the coupling between both metasurface 


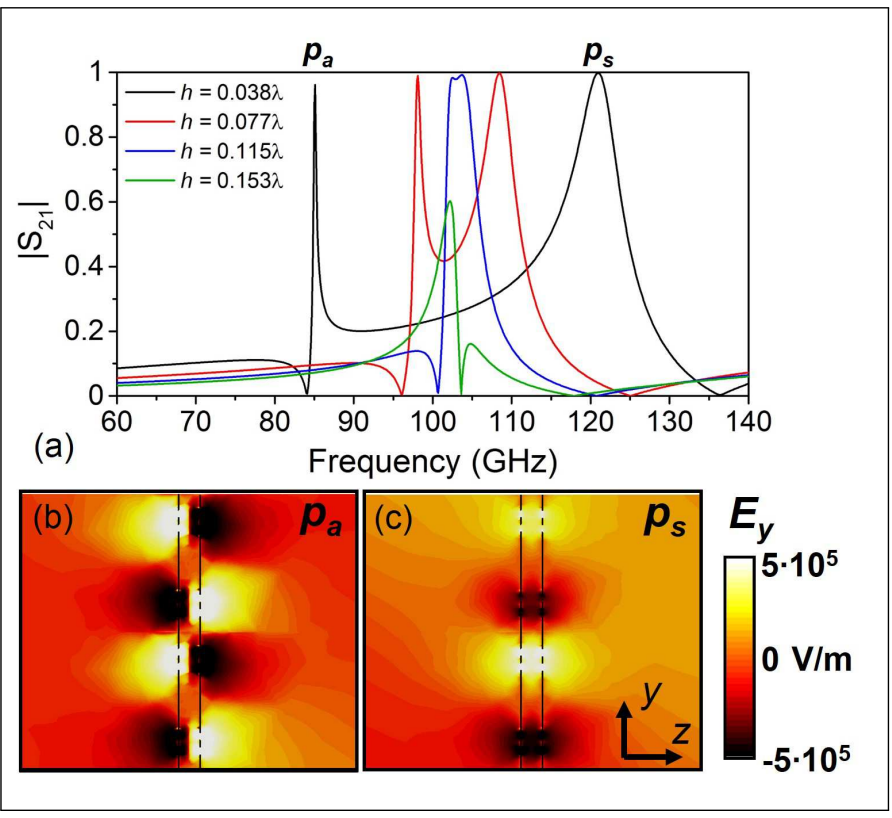

Fig. 4. (a) Transmission results for bi-layer configurations of NB-CSRR - $\mathrm{TM}_{\phi=0^{\circ}}\left(\theta=60^{\circ}\right)$. Electric field distribution of $y$ component: (b) $p_{a}\left(f_{a}=85.1 \mathrm{GHz}\right)$; (c) NB-CSRR $-p_{s}\left(f_{s}=121\right.$ $\mathrm{GHz})$. The thickness $h$, is given in electrical length $\lambda$, being $\lambda$ the effective wavelength within the substrate at $100 \mathrm{GHz}$.

layers. The resulting high transmission peaks can be categorized as two distinct resonant modes with asymmetric and symmetric field distribution. An extensive analysis dealing with this phenomenon occurring at bi- and multi-layer slot-type metallic screens in terms of equivalent circuit approach can be found within the literature, see for instance $[25,26]$. Therefore, here we discuss uniquely the basic concepts.

As it can be seen in Fig. 4, when $h$ is very small the two screens are strongly coupled and a new resonance peak appears. The two peaks show almost total transmission (in the lossless case) and appear quite separated in frequency. Both resonances approach fast to each other as $h$ increases. In fact, at $h=0.153 \lambda$ $\mathrm{mm}$ a mutual cancellation occurs due to a mode hybridization leading to a strong reduction of the transmission level (the incident wave is strongly reflected) for the lossless case. Figure 4 (c) and (d) shows the field distribution for the NB-CSRR case for the two highlighted frequencies, $p_{a}$ and $p_{s}$ which correspond to $f_{a}=85.1$ and $f_{s}=121 \mathrm{GHz}$ respectively. It can be clearly noticed that the field distribution at $p_{a}$ corresponds to an asymmetric mode excitation due to the internal coupling between the two metallic screens. In contrast, the field distribution at $p_{s}$ denotes a symmetric mode that is mainly related to the resonance of the isolated NB-CSRR elements plus the coupling of the neighboring elements which act as loads leading to a slightly different frequency with respect to the mono-layer case.

\section{EXPERIMENT}

In order to experimentally demonstrate the above presented features, a stacked NB-CSRR metasurface prototype was fabricated by means of photolithography techniques. The substrate used was a cyclic olefin copolymer (COC) film which is a flexible material and has low loss and low dispersion at millimeter-wave and $\mathrm{THz}$ frequencies. The substrate has a constant permittivity

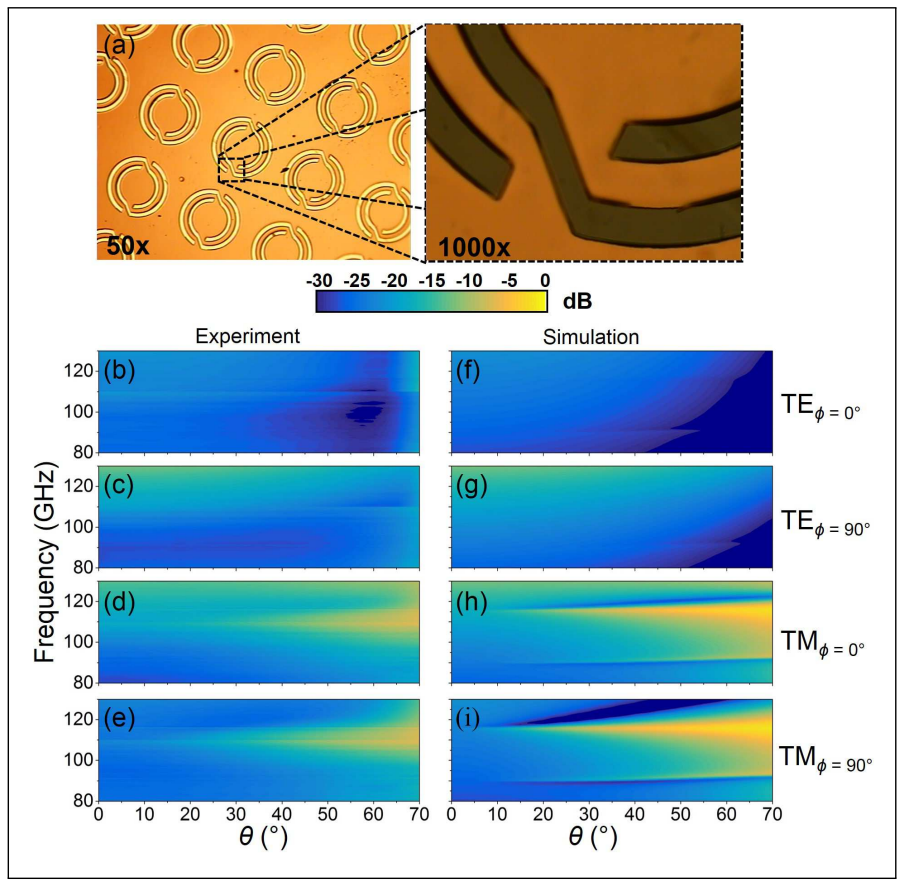

Fig. 5. a) Microscope pictures of the fabricated prototype. Transmission results for NB-CSRR bi-layer configurations. (b) Experiment - $\mathrm{TE}_{\phi=0^{\circ}}$. (c) Experiment - $\mathrm{TE}_{\phi=90^{\circ}}$. (d) Experiment $\mathrm{TM}_{\phi=0^{\circ}}$. (e) Experiment - $\mathrm{TM}_{\phi=90^{\circ}}$. (f) Simulation - $\mathrm{TE}_{\phi=0^{\circ}}$. (g) Simulation - $\mathrm{TE}_{\phi=90^{\circ}}$. (h) Simulation - $\mathrm{TM}_{\phi=0^{\circ}}$. (i) Simulation $\mathrm{TM}_{\phi=90^{\circ}}$.

$\varepsilon_{S}=2.35$, a loss tangent $\tan \delta=0.001$ and a thickness $h=0.1 \mathrm{~mm}$. The metal sheets were fabricated on Copper with a thickness of $1.5 \mu \mathrm{m}$ and nominal conductivity $\sigma_{\mathrm{Cu}}=5.8 \times 10^{7} \mathrm{~S} / \mathrm{m}$. Microscope pictures of the fabricated prototype with 50x and 1000x magnification are shown in Fig. 5(a). The same photo-mask is used for the two metasurface layers leading to a rotation of $180^{\circ}$ between them [see inset in Fig. 3(b)]. To corroborate the validity of the experiment, unit cell CST simulations were conducted with the material properties described above.

The measurements were performed using an ABMillimetre $^{\mathrm{TM}}$ [27] vector network analyzer whose signal generation and detection frequency range covers from tens of $\mathrm{GHz}$ up to $1 \mathrm{THz}$. The VNA was used along with a quasi-optical bench, that comprises transmitter and receiver corrugated horn antennas for the beam generation/detection and a system of elliptical mirrors that focus the beam onto the sample. The sample was mounted on a rotary platform to vary the incidence angle of the incident wave. To switch between polarization states (TM or TE), the whole antenna system was rotated $90^{\circ}$. Frequency and angular sweeps were conducted to fully characterize the spectral and angular response of the NB-CSRR metasurface. To this end, measurements were done from 80 to $130 \mathrm{GHz}$ with a step of $25 \mathrm{MHz}$, and from 0 to $70^{\circ}$ with a step of $2.5^{\circ}$. The results were post-processed by applying a Butterworth filter to reduce undesired reflections.

Figure 5 shows the experimental and simulated transmission results for the fabricated NB-CSRR prototype considering both $\mathrm{TM}$ and TE polarization in the two principal planes. As it can be seen there, measurements and simulations show a qualitative good agreement. The transmission band in the experiment appears at lower frequencies than in the simulation and the 
amplitude is somewhat reduced. It can be also observed that the asymmetrical mode resonance is not fully recorded in the measurement results. It should be taken into account that due to its high quality factor this resonance is very sensitive to losses and fabrication tolerances, to the extent that a small modification between simulated and actual structural values such as longitudinal period, sample alignment, etc., could lead to large differences between numerical and experimental results. Theoretically at larger angles the transmission of both symmetrical and asymmetrical mode resonances approaches to $0 \mathrm{~dB}$ and the resonances bandwidth increases [see Fig. 5 (h) and (i)]. From the previous results, it is obvious that losses are crucial for the overall performance of the NB-CSRR structure.

Concerning the symmetrical mode resonance, it can be seen that the experimental amplitude is below the numerical values. This could be due to additional losses introduced by a non-uniformity of the substrate during fabrication leading to roughness of the metallized parts of the structure and therefore decreasing the effective conductivity of the metal. Additionally, under oblique incidence the focused beam was not effectively illuminating the same amount of elements as for the normal incidence case. All in all, although there is a room for improvement of the experimental results, they show a good qualitative agreement with the numerical simulations.

\section{CONCLUSION}

In this work, a NB-CSRR metasurface has been numerically and experimentally characterized. Its angular response was evaluated demonstrating a very interesting feature in which transmission under normal incidence was forbidden while for oblique incidence under TM polarization high transmission bands were achieved. In addition, the implications of adding a secondary metasurface were also discussed in terms of the excitation of an asymmetrical mode due to the coupling between longitudinally placed metallic screens. For the lossless case, total transmission and narrowband peaks were recorded that could be effectively used for free-space sensing applications. Nevertheless, in the experimental results it was found that the losses destroy the asymmetric mode and decrease considerably the transmission amplitude of the symmetric mode resonance. Nevertheless the experimental results are qualitatively in good agreement with the numerical results. The structure presented here could be used in complex antenna systems where angular and frequency filtering is required as well as for grazing angle sensing devices within the millimeter and $\mathrm{THz}$ frequency ranges.

Funding. This work was funded by the Spanish Ministerio de Economía y Competitividad with project TEC2014-51902-C2-2-R. P. R.-U. acknowledges funding by Public University of Navarra via a pre-doctoral scholarship. M.B. acknowledges funding by the Spanish Ministerio de Economía y Competitividad with contract RYC-2011- 08221.

Acknowledgement. The authors are grateful to Adrián GómezTorrent for help in the fabrication of samples.

\section{REFERENCES}

1. J. B. Pendry, A. J. Holden, D. J. Robbins, and W. J. Stewart, "Magnetism from conductors and enhanced nonlinear phenomena," IEEE Trans. Microw. Theory Tech. 47, 2075-2084 (1999).

2. D. Schurig, J. J. Mock, B. J. Justice, S. A. Cummer, J. B. Pendry, A. F. Starr, and D. R. Smith, "Metamaterial electromagnetic cloak at microwave frequencies," Science 314, 977-980 (2006).
3. V. G. Veselago, "The electrodynamics of substances with simultaneously negative values of $\varepsilon$ and $\mu$," Sov. Phys. Uspekhi 10, 509-514 (1968).

4. R. Marqués, F. Medina, and R. Rafii-El-Idrissi, "Role of bianisotropy in negative permeability and left-handed metamaterials," Phys. Rev. B 65, 144440 (2002).

5. R. Marqués, J. D. Baena, M. Beruete, F. Falcone, T. Lopetegi, M. Sorolla, F. Martín, and J. Garcia, "Ab initio analysis of frequency selective surfaces based on conventional and complementary split ring resonators," J. Opt. A: Pure Appl. Opt. 7, S38 (2005).

6. J. D. Baena, J. Bonache, F. Martin, R. M. Sillero, F. Falcone, T. Lopetegi, M. A. G. Laso, J. Garcia-Garcia, I. Gil, M. Flores Portillo, and M. Sorolla, "Equivalent-circuit models for split-ring resonators and complementary split-ring resonators coupled to planar transmission lines," IEEE Trans. Microw. Theory Tech. 53, 1451-1461 (2005).

7. M. Beruete, M. Sorolla, R. Marqués, J. D. Baena, and M. Freire, "Resonance and cross-polarization effects in conventional and complementary split ring resonator periodic screens," Electromagnetics 26, 247-260 (2006).

8. F. Falcone, T. Lopetegi, M. A. G. Laso, J. D. Baena, J. Bonache, M. Beruete, R. Marqués, F. Martín, and M. Sorolla, "Babinet principle applied to the design of metasurfaces and metamaterials," Phys. Rev. Lett. 93, 197401 (2004).

9. M. Aznabet, M. Navarro-Cía, S. Kuznetsov, A. Gelfand, N. Fedorinina, Y. G. Goncharov, M. Beruete, O. El Mrabet, and M. Sorolla, "Polypropylene-substrate-based srr-and csrr-metasurfaces for submillimeter waves," Opt. Express 16, 18312-18319 (2008).

10. Z. Jakšić, S. Vuković, J. Matovic, and D. Tanasković, "Negative refractive index metasurfaces for enhanced biosensing," Materials 4, 1-36 (2011).

11. C. L. Holloway, E. F. Kuester, J. A. Gordon, J. O'Hara, J. Booth, and D. R. Smith, "An overview of the theory and applications of metasurfaces: The two-dimensional equivalents of metamaterials," IEEE Antennas Propagat. Mag. 54, 10-35 (2012).

12. A. V. Kildishev, A. Boltasseva, and V. M. Shalaev, "Planar photonics with metasurfaces," Science 339, 1232009 (2013).

13. N. Yu, P. Genevet, M. A. Kats, F. Aieta, J.-P. Tetienne, F. Capasso, and Z. Gaburro, "Light propagation with phase discontinuities: generalized laws of reflection and refraction," Science 334, 333-337 (2011).

14. A. Silva, F. Monticone, G. Castaldi, V. Galdi, A. Alù, and N. Engheta, "Performing mathematical operations with metamaterials," Science $\mathbf{3 4 3}$, 160-163 (2014).

15. C. Pfeiffer, C. Zhang, V. Ray, L. J. Guo, and A. Grbic, "High performance bianisotropic metasurfaces: asymmetric transmission of light," Phys. Rev. Lett. 113, 023902 (2014).

16. B. Orazbayev, N. M. Estakhri, M. Beruete, and A. Alù, "Terahertz carpet cloak based on a ring resonator metasurface," Phys. Rev. B 91, 195444 (2015).

17. A. Poddubny, I. lorsh, P. Belov, and Y. Kivshar, "Hyperbolic metamaterials," Nat. Photonics 7, 948-957 (2013).

18. V. A. Fedotov, J. Wallauer, M. Walther, M. Perino, N. Papasimakis, and N. I. Zheludev, "Wavevector selective metasurfaces and tunnel vision filters," Light Sci. Appl. 4, e306 (2015).

19. N. Born, I. Al-Naib, C. Jansen, R. Singh, J. V. Moloney, M. Scheller, and M. Koch, "Terahertz metamaterials with ultrahigh angular sensitivity," Adv. Opt. Mater. 3, 642-645 (2015).

20. P. Aguilà, S. Zuffanelli, G. Zamora, F. Paredes, F. Martín, and J. Bonache, "Front-to-back ratio improvement of printed antennas based on electrically small resonators for microwave presence detectors," Electron. Lett. 51, 836-837 (2015).

21. J. Naqui, M. Durán-Sindreu, and F. Martín, "Modeling split-ring resonator (srr) and complementary split-ring resonator (csrr) loaded transmission lines exhibiting cross-polarization effects," IEEE Antennas Wireless Propag. Lett. 12, 178-181 (2013).

22. CST Microwave Studio (2017), https://www.cst.com/products/cstmws.

23. M. Navarro-Cía, S. A. Kuznetsov, M. Aznabet, M. Beruete, F. Falcone, and M. S. Ayza, "Route for bulk millimeter wave and terahertz metamaterial design," IEEE J. Quantum Electron. 47, 375-385 (2011).

24. R. Ortuño, C. García-Meca, F. Rodríguez-Fortuño, J. Martí, and 
A. Martínez, "Role of surface plasmon polaritons on optical transmission through double layer metallic hole arrays," Phys. Rev. B 79, 075425 (2009).

25. V. Torres, F. Mesa, M. Navarro-Cía, R. Rodríguez-Berral, M. Beruete, and F. Medina, "Accurate circuit modeling of fishnet structures for negative-index-medium applications," IEEE Trans. Microw. Theory Tech. 64, 15-26 (2016).

26. C. S. Kaipa, A. B. Yakovlev, F. Medina, F. Mesa, C. A. Butler, and A. P. Hibbins, "Circuit modeling of the transmissivity of stacked twodimensional metallic meshes," Opt. Express 18, 13309-13320 (2010).

27. AB Millimetre-Company Specialized in MVNA manufacturing, http: //www.abmillimetre.com. 